$01 ; 11$

\title{
Решение электродинамической задачи для микрополосковой излучающей структуры с киральной подложкой
}

\author{
(C) М.А. Бузова ${ }^{1}$, Д.С. Клюев ${ }^{2,}$, М.А. Минкин ${ }^{1}$, А.М. Нещерет ${ }^{1}$, \\ Ю.В. Соколова ${ }^{2}$ \\ ${ }^{1}$ Концерн „Автоматика“, Москва, Россия \\ ${ }^{2}$ Поволжский государственный университет телекоммуникаций \\ и информатики, Самара, Россия \\ ฯ E-mail: klyuevd@yandex.ru
}

Поступило в Редакцию 8 декабря 2017 г.

Приведено решение электродинамической задачи для микрополосковой излучающей структуры с подложкой из кирального метаматериала методом сингулярного интегрального представления поля, которое в свою очередь сведено к сингулярному интегральному уравнению с особенностью типа Коши относительно продольной составляющей поверхностной плотности тока. Представлены графики распределения тока при различных типах подложек и значениях параметра киральности подложки.

DOI: $10.21883 /$ PJTF.2018.11.46200.17147

Одной из важных задач в антенной технике в настоящее время является сокращение массогабаритных показателей антенн при приемлемых электрических характеристиках. Безусловный прорыв в данном направлении в области СВЧ был совершен при создании микрополосковых антенн (МПА), представляющих собой подложку из высокочастотного диэлектрика с расположенным на ней планарным излучателем. Дальнейший прогресс в рассматриваемой области техники, как показали результаты многочисленных исследований, может быть обеспечен на основе использования в конструкции МПА искусственных композитных структур - метаматериалов [1-3], обладающих целенаправленно формируемыми электрофизическими характеристиками и позволяющих значительно улучшить электрические и массогабаритные характеристики МПА. Для анализа таких структур будем использовать метод сингулярного интегрального представления (СИП) поля [4]. В на- 


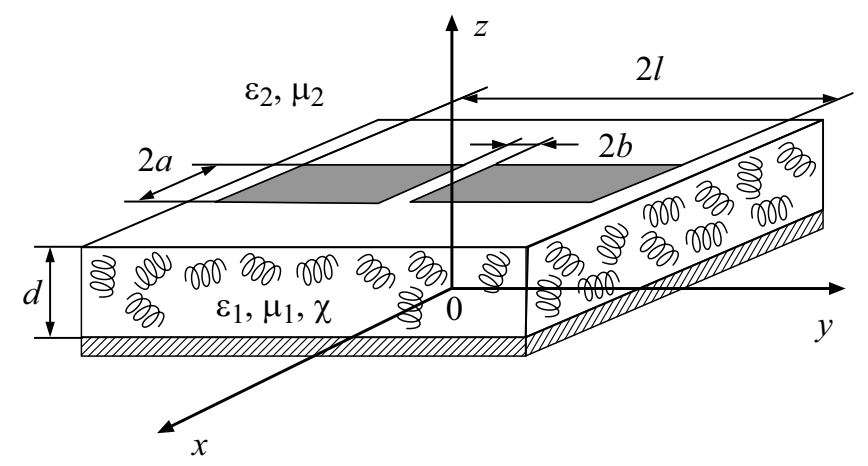

Рис. 1. Геометрия микрополосковой излучающей структуры с киральной подложкой.

стоящей работе приведено решение электродинамической задачи для микрополосковой излучающей структуры с подложкой из кирального метаматериала методом СИП поля.

Рассмотрим микрополосковую излучающую структуру (рис. 1), представляющую собой подложку из кирального метаматериала [5] с макроскопическими параметрами $\varepsilon_{1}, \mu_{1}, \chi$, металлизированную с нижней стороны и имеющую конечную толщину $d$, на которой расположена бесконечно тонкая и идеально проводящая пластина конечных размеров, длина которой составляет $2 l$, а ширина - $2 a$. Над проводящей пластиной расположено диэлектрическое полупространство с макроскопическими параметрами $\varepsilon_{2}, \mu_{2}$. Киральный метаматериал (киральная среда) был выбран из-за его „особых“ электродинамических свойств, одним из которых является способность поворота плоскости поляризации нормально падающих электромагнитных волн. Однако никаких ограничений на применение в качестве материала подложки других типов метаматериалов предложенный метод не накладывает.

Ширина излучателя считается достаточно малой, так что поперечной составляющей поверхностной плотности тока $\eta_{x}$ можно пренебречь: $\eta=\left(0, \eta_{y}\right)$. В зазор излучателя подключен сторонний источник ЭДС таким образом, что функция поверхностной плотности тока является непрерывной как на поверхности излучателя, так и в области зазора. Кроме того, предполагается также, что тангенциальная составляющая

6 Письма в ЖТФ, 2018, том 44, вып. 11 
электрического поля, создаваемого сторонним источником ЭДС, имеет только одну компоненту: $\mathbf{E}^{e x t}=\left\{0, E_{y}^{e x t}, 0\right\}$. Наконец, на поверхности излучателя предполагается выполнение следующих граничных условий:

$$
\begin{gathered}
\eta_{y}(x,-l)=\eta_{y}(x,+l)=0, \\
E_{\tau}(x, y)=0, \quad x \in[-a, a], \quad y \in[-l,-b] \cup[b, l], \\
E_{\tau}(x, y)=-E_{\tau}^{e x t}, \quad x \in[-a, a], \quad y \in[-b, b] .
\end{gathered}
$$

Разложим векторы напряженности электрического и магнитного полей, а также вектор поверхностной плотности тока в интеграл Фурье [4]. На поверхности излучателя фурье-образ тангенциальной составляющей напряженности электрического поля $\mathbf{T}_{\tau}=\left\{T_{x}, T_{y}\right\}$ связан с фурье-образом поверхностной плотности тока $\mathbf{F}=\left\{F_{x}, F_{y}\right\}$ через матрицу поверхностных импедансов $[Z][4]$

$$
\left[\begin{array}{l}
T_{y} \\
T_{x}
\end{array}\right]=\left[\begin{array}{ll}
Z_{11} & Z_{12} \\
Z_{21} & Z_{22}
\end{array}\right]\left[\begin{array}{l}
F_{y} \\
F_{x}
\end{array}\right]
$$

Для нахождения данной матрицы сначала получают обратную ей матрицу поверхностных адмитансов, которая в свою очередь выражается через матрицы входных адмитансов каждого слоя. Более подробно методика определения матрицы поверхностных импедансов границы раздела диэлектрик-киральная среда приведена в работах [6,7]. Выражения элементов матрицы входных адмитансов для кирального слоя на основе лево- и правосторонних элементов имеют следующий вид:

$$
\begin{gathered}
Y_{11}^{(1)}(\beta, h)=\frac{a(h)\left[i \omega \varepsilon_{0} \varepsilon_{1} v_{p}(\beta, h)-Y_{12}^{(1)}(\beta, h) w_{p}(\beta, h)\right]}{ \pm\left(\sin \left(\gamma_{R} z\right)+\sin \left(\gamma_{L} z\right)\right)}, \\
Y_{12}^{(1)}(\beta, h)=\frac{w_{m}(\beta, h)}{i \omega \mu_{0} \mu_{1} v_{m}(\beta, h)}, \quad Y_{21}^{(1)}(\beta, h)=\frac{a(h) Y_{22}^{(1)}(\beta, h) w_{p}(\beta, h)}{\mp\left(\sin \left(\gamma_{R} z\right)+\sin \left(\gamma_{L} z\right)\right)}, \\
Y_{22}^{(1)}(\beta, h)=\frac{\sin \left(\gamma_{R} z\right)-\sin \left(\gamma_{L} z\right)}{i \omega \mu_{0} \mu_{1} v_{m}(\beta, h) a(h)},
\end{gathered}
$$

где $k=\omega \sqrt{\mu_{0} \varepsilon_{0}}=2 \pi / \lambda-$ волновое число; $i=\sqrt{-1}-$ мнимая единица,

$$
a(h)=\frac{1}{k^{2} \varepsilon_{1} \mu_{1}+k^{2} \chi^{2}-h^{2}}\left(1-\frac{4 k^{4} \chi^{2} \varepsilon_{1} \mu_{1}}{\left(k^{2} \varepsilon_{1} \mu_{1}+k^{2} \chi^{2}-h^{2}\right)^{2}}\right)^{-1}
$$

Письма в ЖТФ, 2018, том 44, вып. 11 


$$
\begin{gathered}
\xi(h)=\frac{2 k \chi}{k^{2} \varepsilon_{1} \mu_{1}+k^{2} \chi^{2}-h^{2}}, \\
s(h)=1-\frac{2 k^{2} \chi^{2}}{k^{2} \varepsilon_{1} \mu_{1}+k^{2} \chi^{2}-h^{2}}, \\
q(h)=k \chi-\frac{2 k^{3} \chi \varepsilon_{1} \mu_{1}}{k^{2} \varepsilon_{1} \mu_{1}+k^{2} \chi^{2}-h^{2}}, \\
v_{p}(\beta, h)=-s(h)\left[ \pm\left(\gamma_{R} \cos \left(\gamma_{R} z\right)+\gamma_{L} \cos \left(\gamma_{L} z\right)\right)\right] \\
+\zeta(h) \beta h\left(\sin \left(\gamma_{R} z\right)+\sin \left(\gamma_{L} z\right)\right), \\
v_{m}(\beta, h)=-s(h)\left(\gamma_{R} \cos \left(\gamma_{R} z\right)-\gamma_{L} \cos \left(\gamma_{L} z\right)\right) \\
\pm \zeta(h) \beta h\left(\sin \left(\gamma_{R} z\right)-\sin \left(\gamma_{L} z\right)\right), \\
w_{p}(\beta, h)=-\beta h\left[ \pm\left(\sin \left(\gamma_{R} z\right)+\sin \left(\gamma_{L} z\right)\right)\right] \\
\mp q(h)\left( \pm\left(\gamma_{R} \cos \left(\gamma_{R} z\right)+\gamma_{L} \cos \left(\gamma_{L} z\right)\right)\right),
\end{gathered}
$$

$w_{m}(\beta, h)=\beta h\left(\sin \left(\gamma_{R} z\right)-\sin \left(\gamma_{L} z\right)\right) \pm q(h)\left(\gamma_{R} \cos \left(\gamma_{R} z\right)-\gamma_{L} \cos \left(\gamma_{L} z\right)\right)$, $\gamma_{R}=\sqrt{k^{2}(n+\chi)^{2}-\beta^{2}-h^{2}}, \quad \gamma_{L}=\sqrt{k^{2}(n-\chi)^{2}-\beta^{2}-h^{2}}, n=\sqrt{\varepsilon_{1} \mu_{1}}$.

В этих выражениях верхние знаки соответствуют киральной структуре на основе правосторонних элементов, а нижние - на основе левосторонних. Элементы матрицы входных адмитансов диэлектрического слоя приведены в [4].

Применяя к данной матрице обратное преобразование Фурье и учитывая при этом, что поверхностная плотность электрического тока $\eta$ отлична от нуля только на поверхности пластины $(z=d, x \in[-a, a], y \in[-l, l])$, получаем следующее выражение для напряженности стороннего поля:

$$
E_{y}^{e x t}(x, y, z=d)=\int_{-a}^{a} \int_{-l}^{l} \eta_{y}\left(x^{\prime}, y^{\prime}\right) Z^{\Sigma}\left(x^{\prime}, y^{\prime}, x, y\right) d x^{\prime} d y^{\prime},
$$

где

$$
Z^{\Sigma}\left(x^{\prime}, y^{\prime}, x, y\right)=\frac{1}{4 \pi^{2}} \int_{-\infty}^{\infty} \int_{-\infty}^{\infty} Z_{11}(\beta, h) e^{-i \beta\left(x-x^{\prime}\right)} e^{-i h\left(y-y^{\prime}\right)} d \beta d h .
$$

$6^{*}$ Письма в ЖТФ, 2018, том 44, вып. 11 
Следует отметить, что несобственный интеграл в (4) является расходящимся. Устраняя данную расходимость путем суммирования и вычитания асимптотического сомножителя $Z_{11}^{\infty}$ в приведенном выше выражении, а также предполагая, что поперечная вариация продольного распределения поверхностной плотности тока описывается квазистатическим приближением [4]: $\eta_{y}\left(x^{\prime}, y^{\prime}\right)=f\left(y^{\prime}\right) / \sqrt{1-\left(x^{\prime} / a\right)^{2}}$, где $f\left(y^{\prime}\right)-$ функция, описывающая продольное распределение поверхностной плотности тока, и проводя ряд дополнительных преобразований, получим выражение СИП электрического поля. Подробное описание методики получения СИП приведено в [4].

Подставляя граничные условия (1) в СИП (4), получим сингулярное интегральное уравнение с особенностью Коши относительно неизвестной функции $f\left(y^{\prime}\right)$

$$
\begin{aligned}
-E_{y}^{e x t}(0, y, d)= & -\frac{a}{4 \pi} \int_{-l}^{l} f^{\prime}\left(y^{\prime}\right) \int_{-\infty}^{\infty} \int_{-\infty}^{\infty} J_{0}(\beta a) \frac{\Delta Z_{11}(\beta, h)}{i h} e^{-i h\left(y-y^{\prime}\right)} d \beta d h d y^{\prime} \\
& -\frac{C_{\varepsilon, \mu, \chi}}{\pi} \int_{-l}^{l} \frac{f^{\prime}\left(y^{\prime}\right)}{y^{\prime}-y} d y^{\prime}
\end{aligned}
$$

где $\Delta Z_{11}(\beta, h)=Z_{11}(\beta, h)-Z_{11}^{\infty}(h) ; Z_{11}^{\infty}(h)-$ это $Z_{11}(\beta, h)$ при $|h| \rightarrow \infty$,

$$
\begin{gathered}
Z_{11}(\beta, h) \underset{|h| \rightarrow \infty}{\longrightarrow} Z_{11}^{\infty}(h)=C_{\varepsilon, \mu, \chi}|h|, \\
C_{\varepsilon, \mu, \chi}=\frac{i \omega \mu_{0}\left(\mu_{1}+\mu_{2}\right)}{\left(k^{2}\left(\varepsilon_{1}+\varepsilon_{2}\right)\left(\mu_{1}+\mu_{2}\right)-(k \chi)^{2}\right)} .
\end{gathered}
$$

В результате решения приведенного выше уравнения определяется функция, описывающая продольное распределение поверхностной плотности тока. Методы решения сингулярных интегральных уравнений, подобных (5), подробно описаны в [4]. В свою очередь токовая функция определяется в соответствии с выражением

$$
I_{y}\left(y^{\prime}\right)=\int_{-a}^{a} \eta_{y}\left(x^{\prime}, y^{\prime}\right) d x=\int_{-a}^{a} \frac{f\left(y^{\prime}\right)}{\sqrt{1-\left(x^{\prime} / a\right)^{2}}} d x=a \pi f\left(y^{\prime}\right) .
$$

Письма в ЖТФ, 2018, том 44, вып. 11 


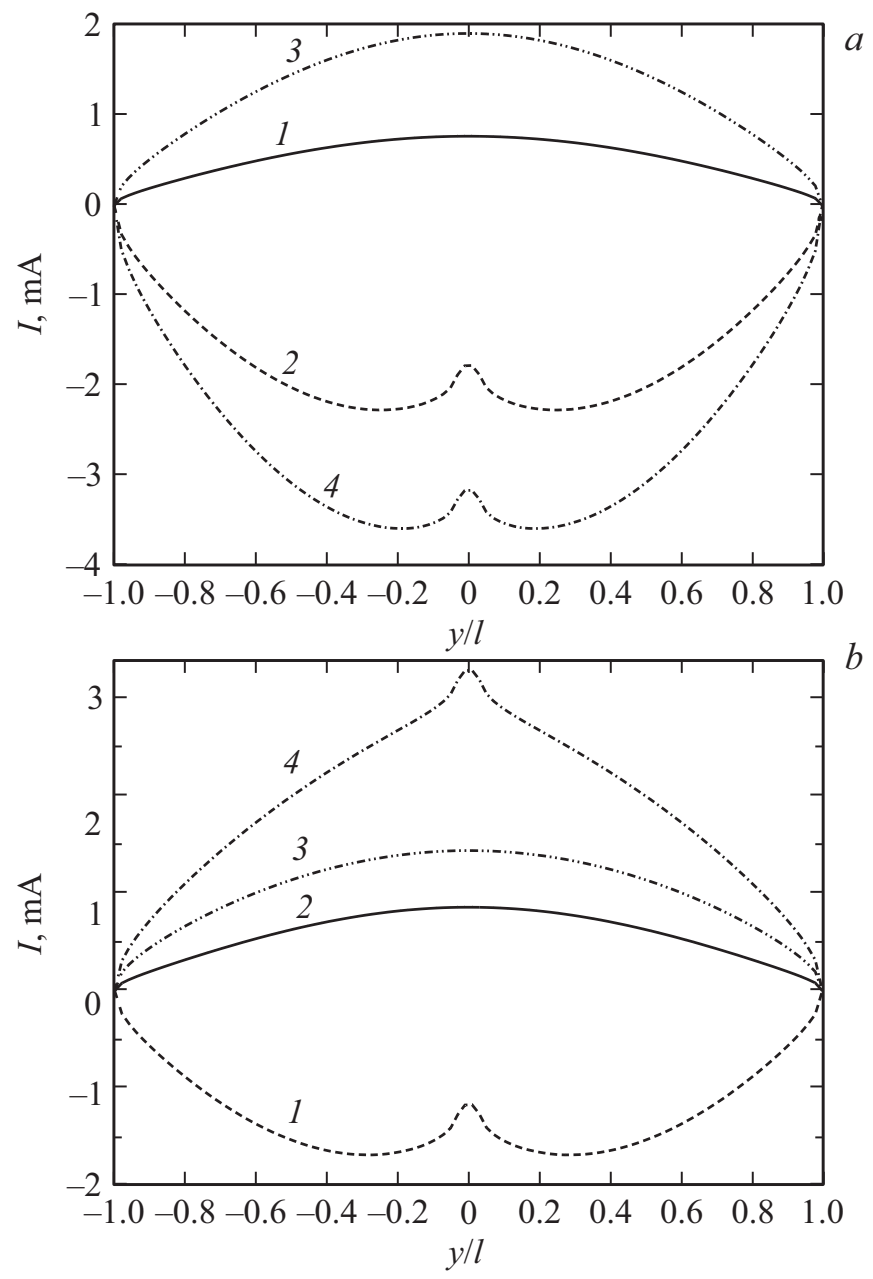

Рис. 2. Распределение тока на поверхности излучателя, расположенного на киральной подложке при параметре киральности $\chi=0.15(a)$ и $0.5(b) .1$ и 2 - соответственно действительная и мнимая части тока в случае подложки на основе правосторонних элементов, 3 и $4-$ соответственно действительная и мнимая части тока в случае подложки на основе левосторонних элементов.

Письма в ЖТФ, 2018, том 44, вып. 11 
На рис. 2 представлены графики распределения тока по излучателю, расположенному на подложке из кирального метаматериала на основе лево- и правовинтовых спиралей со следующими параметрами: толщина $d=0.1 \lambda, \varepsilon_{1}=\varepsilon_{2}=1, \mu_{1}=\mu_{2}=1, \chi=0.15$ (рис. $\left.2, a\right)$ и 0.5 (рис. $2, b$ ). При этом геометрические параметры излучателя равны: длина $2 l=0.5 \lambda$, ширина $2 a=0.05 \lambda$, ширина зазора $2 b=0.02 \lambda$, где $\lambda-$ длина волны.

Таким образом, в работе приведена методика нахождения распределения тока по микрополосковой излучающей структуре с киральной подложкой. Установлено, что тип подложки существенно влияет на токовую функцию, причем чем больше значение параметра киральности $\chi$, тем более существенны различия.

\section{Список литературы}

[1] Вендик И.Б., Вендик О.Г. // ЖТФ. 2013. Т. 83. В. 1. С. 3-28.

[2] Слюсар В.И. // Электроника: НТБ. 2009. № 7. С. 10-19.

[3] Шорохова Е.А., Манахова М.С. // Антенны. 2012. В. 12 (187). С. 11-31.

[4] Дементьев А.Н., Клюев Д.С., Неганов В.А., Соколова Ю.В. Сингулярные и гиперсингулярные интегральные уравнения в теории зеркальных и полосковых антенн. М.: Радиотехника, 2015. 216 с.

[5] Неганов B.A., Осипов О.В. Отражающие, волноведущие и излучающие структуры с киральными элементами. М.: Радио и связь, 2006. 280 с.

[6] Клюев Д.С., Нещерет А.М., Осипов О.В., Почепчов А.О. // Успехи соврем. радиоэлектроники. 2015. № 11. С. 67-72.

[7] Нещерет А.М. // Радиотехника. 2016. № 4. С. 118-126. 